\title{
The Transcriptome of Fusarium graminearum During the Infection of Wheat
}

\author{
Erik Lysøe, ${ }^{1}$ Kye-Yong Seong, ${ }^{2}$ and H. Corby Kistler ${ }^{2,3}$ \\ ${ }^{1}$ Bioforsk-Norwegian Institute of Agricultural and Environmental Research, Ås, Norway; ${ }^{2}$ Department of Plant Pathology, \\ University of Minnesota, St. Paul 55108, U.S.A.; ${ }^{3}$ United States Department of Agriculture-Agricultural Research Service, \\ Cereal Disease Laboratory, St. Paul, MN 55108, U.S.A.
}

Submitted 4 March 2011. Accepted 5 May 2011.

\begin{abstract}
Fusarium graminearum causes head blight disease in wheat and barley. To help understand the infection process on wheat, we studied global gene expression of $F$. graminearum in a time series from 24 to $196 \mathrm{~h}$ after inoculation, compared with a noninoculated control. The infection was rapid and, after $48 \mathrm{~h}$, over 4,000 fungal genes were expressed. The number of genes expressed increased over time up to $96 \mathrm{~h}(>8,000$ genes), and then declined at the 144- and 192-h post-inoculation time points. After subtraction of genes found expressed on complete medium, during carbon or nitrogen starvation, and on barley, only 355 were found exclusively expressed in wheat, mostly genes with unknown function (72.6\%). These genes were mainly found in single-nucleotide polymorphism-enriched islands on the chromosomes, suggesting a higher evolutionary selection pressure. The annotated genes were enriched in functional groups predicted to be involved in allantoin and allantoate transport, detoxification, nitrogen, sulfur and selenium metabolism, secondary metabolism, carbohydrate metabolism, and degradation of polysaccharides and ester compounds. Several putative secreted virulence factors were also found expressed in wheat.
\end{abstract}

The filamentous fungus Fusarium graminearum sensu stricto (O'Donnell et al. 2004) is one of the most destructive plant pathogens of small grain cereals. It causes Fusarium head blight (FHB) on wheat and barley (also known as scab), and stalk and ear rot disease of corn (Goswami and Kistler 2004). FHB has great economic impact for cereal farmers and industry due to yield and quality losses and production of several harmful mycotoxins such as the trichothecene deoxynivalenol that contaminate the grain, rendering it unsafe for human and livestock consumption. These secondary metabolites are mainly produced in the growing season but also during storage, when environmental conditions are favorable for fungal growth (McMullen et al. 1997). Fusarium toxins are generally thermostable and unaffected by processing techniques (Lauren and Smith 2001).

FHB is a global problem and has, therefore, been intensively researched. The genome of $F$. graminearum has been sequenced, annotated, and compared with other organisms (Cuomo et al. 2007; Ma et al. 2010). A whole-genome microarray has been developed for $F$. graminearum, a useful tool for global gene-

Corresponding author: E. Lysøe; Telephone: +47 92609123; Fax: +47 64946110; E-mail: erik.lysoe@ bioforsk.no

* The $\boldsymbol{e}$-Xtra logo stands for "electronic extra" and indicates that five supplementary tables are published online. expression studies (Guldener et al. 2006). The goal of the present study was to analyze the transcriptome of $F$. graminearum during the infection process of wheat and compare it with published microrarray experiments done during the infection process in barley, as well as cultivation on three media: complete medium, as well as carbon- and nitrogen-starvation media (Guldener et al. 2006). The goal was to elucidate cellular processes specifically involved in wheat infection, as well as common processes found in both wheat and barley. Identifying essential elements of pathogen-host interactions could lead to development of new targets for fungal control. In this study, whole-genome gene expression was performed during the infection process of wheat, at several time points after point inoculation with $F$. graminearum.

\section{RESULTS}

We compared our results with published microarray data from an $F$. graminearum infection series in barley, from the fungus grown in complete medium and under carbon- or nitrogenstarvation conditions (Guldener et al. 2006). CEL files (normalized by robust multichip analysis [RMA]) from these previously published experiments (FG1 and FG2) were obtained from PLEXdb and analyzed together with our data from inoculated wheat. The expression values of all probe sets in all experiments can be found in Supplementary Table S1. During plant infection, there was an increasing number of detected probe sets over time, with a higher number detected in wheat than in barley during all time points, except for the last barley time point, $144 \mathrm{~h}$ after inoculation (hai) (Fig. 1). The cumulative number of expressed probe sets detected at one or more time points during the infection time course was 10,007 in wheat and 7,777 in barley; 2,477 probe sets were detected only in wheat compared with 247 only in barley. Based on the number of probe sets detected at each time point, the infection of wheat was also more rapid than barley. The profile of expressed probe sets in barley was similar to that detected in wheat $24 \mathrm{~h}$ earlier. This is illustrated in Figure 2, where gene expression in barley clusters with earlier wheat data (e.g., barley $24 \mathrm{~h}$ to wheat control, barley $48 \mathrm{~h}$ to wheat $24 \mathrm{~h}$, and so on). Except for this delay, the expression profiles in both wheat and barley were similar and, together, represent two main clusters (early time points and late time points), separate from expression in complete medium as well as $\mathrm{C}$ - and $\mathrm{N}$-starvation media (third main cluster).

To identify genes expressed exclusively in wheat, all probe sets detected at any condition in vitro, in complete medium or $\mathrm{C}$ - and N-starvation media, were first combined. This combined set of 11,979 probe sets was then compared with the 10,007 
A Expressed F. graminearum probesets in wheat and barley
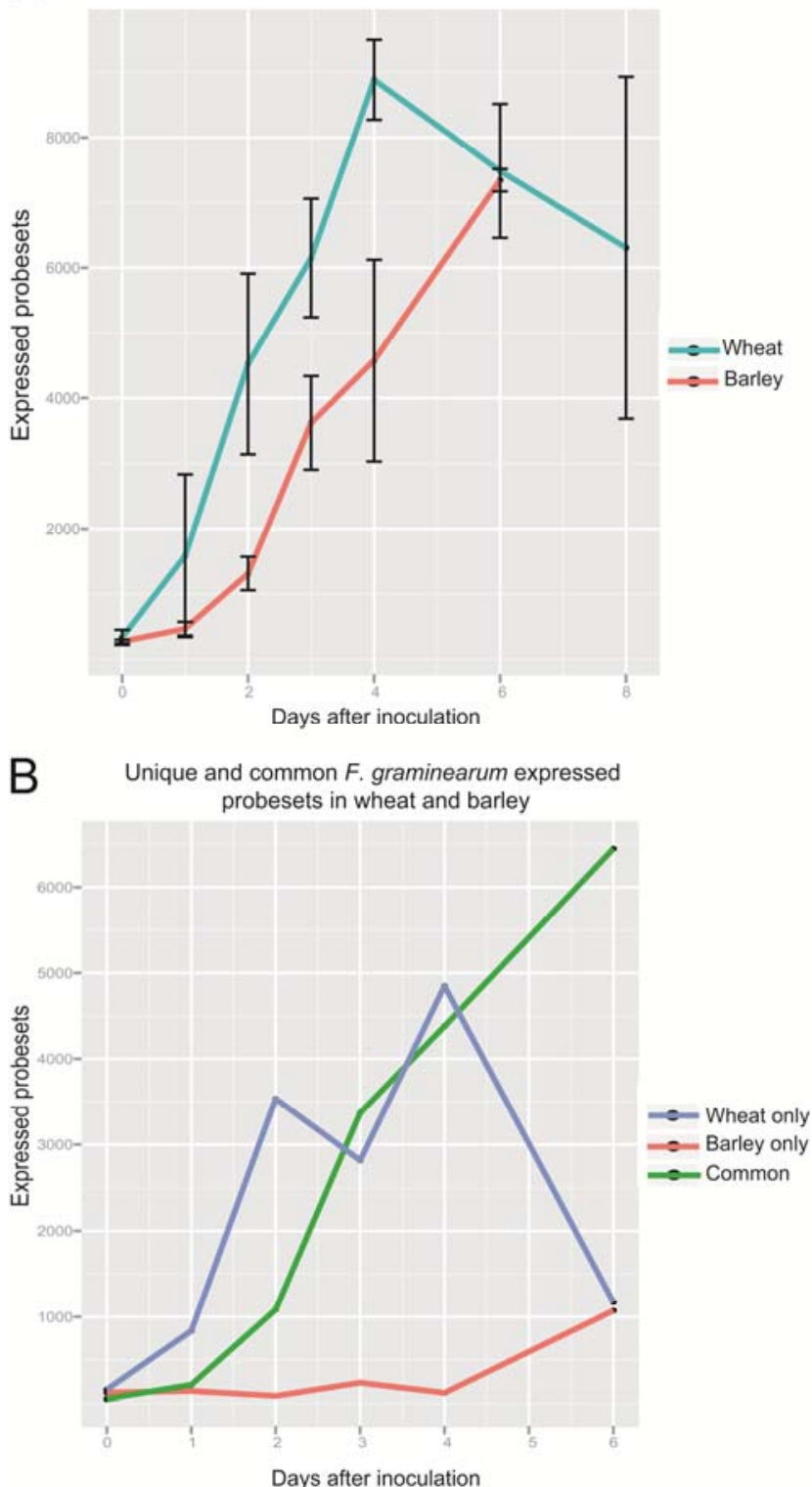

Fig. 1. Number of Fusarium graminearum probe sets detected during infection of wheat or barley. Microarray results were first normalized by robust multichip analysis, and the analysis of data were performed at $P=0.04$. The number of expressed probe sets was noted, and error bars indicate the standard deviation of the three biological replicates. probe sets detected in wheat and 7,777 probe sets detected in barley (Fig. 3; Supplementary Table S2). Only 404 probe sets were detected exclusively in wheat, corresponding to 355 genes annotated in the Munich Information Center for Protein Sequences (MIPS) FGDB database (Mewes et al. 2004). The most frequently observed gene category among those genes expressed exclusively in wheat where unclassified proteins $(258 / 355 ; 72.7 \%)$ (Ruepp et al. 2004). This category was present at a significantly higher percentage $(P=0.005)$ than found in the genome as a whole (Supplementary Table S3). Other functional categories significantly enriched among the 355 genes exclusive to wheat were genes involved in allantoin and allantoate transport $(P=0.001)$, detoxification $(P=0.003)$, nitrogen, sulfur and selenium metabolism $(P=0.006)$, secondary metabolism $(P=0.006)$, carbohydrate metabolism $(P=$ $0.016)$, and degradation of polysaccharides and ester com-

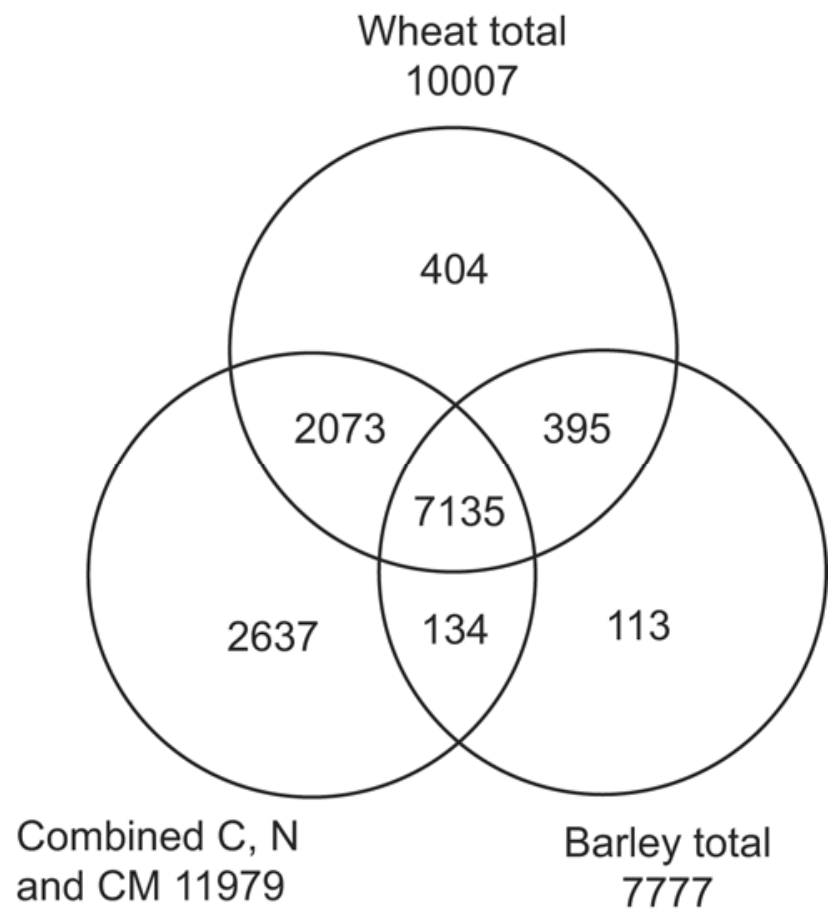

Fig. 3. Venn diagram of probe sets expressed in wheat $(n=10,007)$, barley $(n=7,777)$, and combined complete media and $\mathrm{C}$ and $\mathrm{N}$ starvation $(n=$ $11,979)$, with all probe sets expressed in one or more conditions. The resulting 404 probe sets (355 genes) were detected exclusively in wheat, and 395 probe sets ( 369 genes) were found in both wheat and barley.

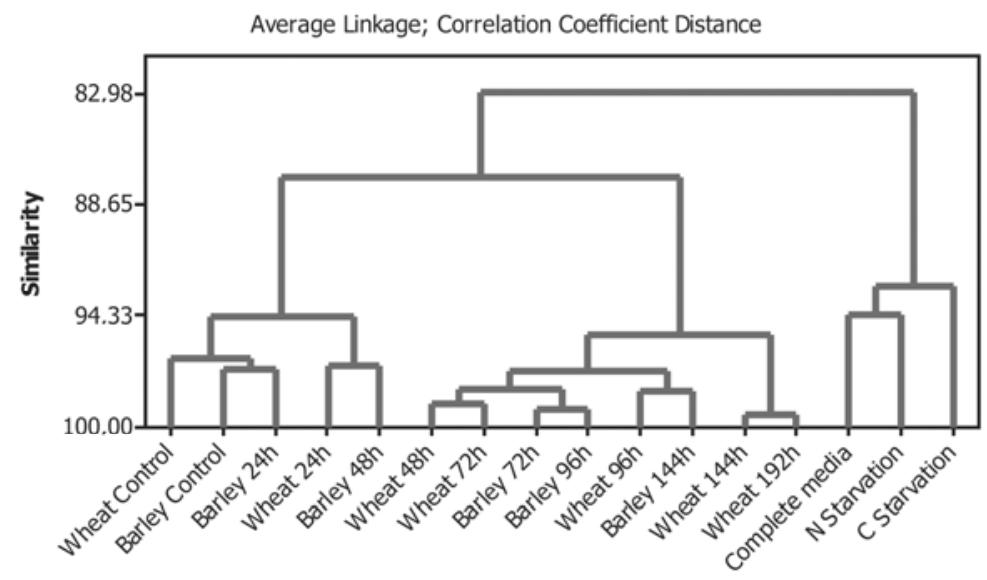

Fig. 2. Similarity dendrogram of Fusarium graminearum expression profiles on wheat, barley, complete media, and C and N starvation. The CEL files were normalized using the "affy" package in R, and the dendrogram shows the hierarchical clustering of the expression pattern of each experiment. 
pounds $(P=0.025)$. In the functional category allantoin and allantoate transport, four of a total of seven genes were suggested to be allantoate permeases. According to MIPS FGDB, the total number of allantoate permeases in the genome is 21 ; therefore, these are enriched in the 355 wheat-specific genes $\left(\chi^{2}, P=2 \mathrm{E}-6\right)$. The polyketide synthases PKS6 (FGSG_08208), PKS8 (FGSG_03340), PKS10 (FGSG_07798), and PKS13 (FGSG_15980) and the KP4 killer toxin ortholog TOX4 (FGSG_10551) were among genes expressed exclusively in wheat. From the 355 wheat-exclusive genes, 28 (7.8\%) had a secretory pathway signal peptide motif compared with the genome as a whole, where $12.8 \%$ of the total number of genes had that motif, which is significantly less than expected $\left(\chi^{2}, P=\right.$ $0.005)$. The timing and expression patterns of genes during infection in wheat are illustrated in Figure 4A and B (Supplementary Table S4a, all genes, and b, wheat only, respectively).

The 395 probe sets expressed in both wheat and barley corresponded to 369 annotated genes. Among the over-represented functional categories of genes expressed here were carbohydrate metabolism, extracellular ester and polysaccharide degradation, polysaccharide binding, disease, virulence and defense and secondary metabolism (Supplementary Table S5). Among the genes for secondary metabolites were those involved in the biosynthesis of the trichothecene mycotoxin deoxynivalenol (Fig. 5), and also predicted genes for several polyketide synthases: PKS5 (FGSG_17677), PKS11 (FGSG_ 01790), PKS14 (FGSG_03964), and PKS15 (FGSG_04588); nonribosomal peptide synthetases NPS9 (FGSG_10990), NPS12 (FGSG_17574), and NPS14 (FGSG_11395) (Fig. 5); and KP4 killer toxin orthologs TOX1 (FGSG_00060), TOX2 (FGSG_00061), and TOX3 (FGSG_00062). One interesting feature was that $43 \%$ of the genes expressed in wheat and barley had a secretory pathway signal peptide motif, which is significantly enriched compared with the genome as a whole, where only $12.8 \%$ genes had that motif $\left(\chi^{2}, P=2.2 \mathrm{E}-16\right)$.

We also compared our gene expression results with the results of Paper and associates (2007), who did several proteomic experiments on wheat heads inoculated with $F$. graminearum. They found that, among the $120 \mathrm{~F}$. graminearum proteins expressed in wheat, the two largest categories are enzymes that act on plant cell walls, and proteases. When compared with our expression data, only seven genes found by proteomic analysis were not found among the expressed genes in wheat or barley (FGSG_00777, FGSG_00806, FGSG_02897, FGSG_03027, FGSG_03143, FGSG_08721, and FGSG_09998). Four genes were found only in wheat (FGSG_00237, related to trichothecene 3-O-acetyltransferase; FGSG_06932, related to adenosine kinase; FGSG_11169, related to $\alpha$-galactosidase; and FGSG 11176, conserved hypothetical protein). The rest (109 genes) were expressed in both wheat and barley. These results support the contention that expression inferred from microarray data is highly concordant with proteomic expression data.

Cuomo and associates (2007) suggested possible virulence factors among predicted secreted proteins specifically expressed in barley and found in single-nucleotide polymorphism (SNP) high-density regions. This included several putative homologs of known virulence factors (Gas1/Gas2 [FG00006], PTTH1 [FG12008], and hydrophobin [FG01831] with homology to cryparin and cerato-ulmin) and possible virulence factors based on function, such as xylanases, acetyl xylan esterases, xylan 1,4- $\beta$-xylosidases, cutinases, pectate lyases, polygalacturonase, secreted proteases, cytochrome P450s, carboxylesterases, glycosyl hydrolases, endoglucanases, and cellulase (See Cuomo and associates [2007], Table S16, for corresponding $F$. graminearum genes). All $45 F$. graminearum genes were found expressed at one or more time points in wheat. Cuomo and associates (2007) also presented 704 genes specific to $F$. grami- nearum, with no homology to any other known protein and having at least one InterPro domain. Of these, 307 were found expressed in wheat and $92 \%$ were characterized as unclassified proteins $(P=2.6 \mathrm{E}-32)$. Of the 355 annotated genes detected exclusively in wheat, 30 belonged to the 704 genes specific to F. graminearum.

Ma and associates (2010) identified 717 transcription factors in $F$. graminearum. Only six were exclusively expressed in wheat (FGSG_11061, FGSG_06699, FGSG_02368, FGSG_ 08753, and FGSG_12006/FGSG_12007), and three were found exclusively expressed in both wheat and barley (FGSG_08080, FGSG_03695, and FGSG_03536 [Tri6]). Of these, only Tri6 showed a high expression rate (48 to $192 \mathrm{~h}$ ) and the other two from wheat and barley were expressed only slightly over background (48 to $192 \mathrm{~h}$ ). The transcription factor genes detected in wheat had inconsistent expression slightly over background in some of the time points (results not shown).
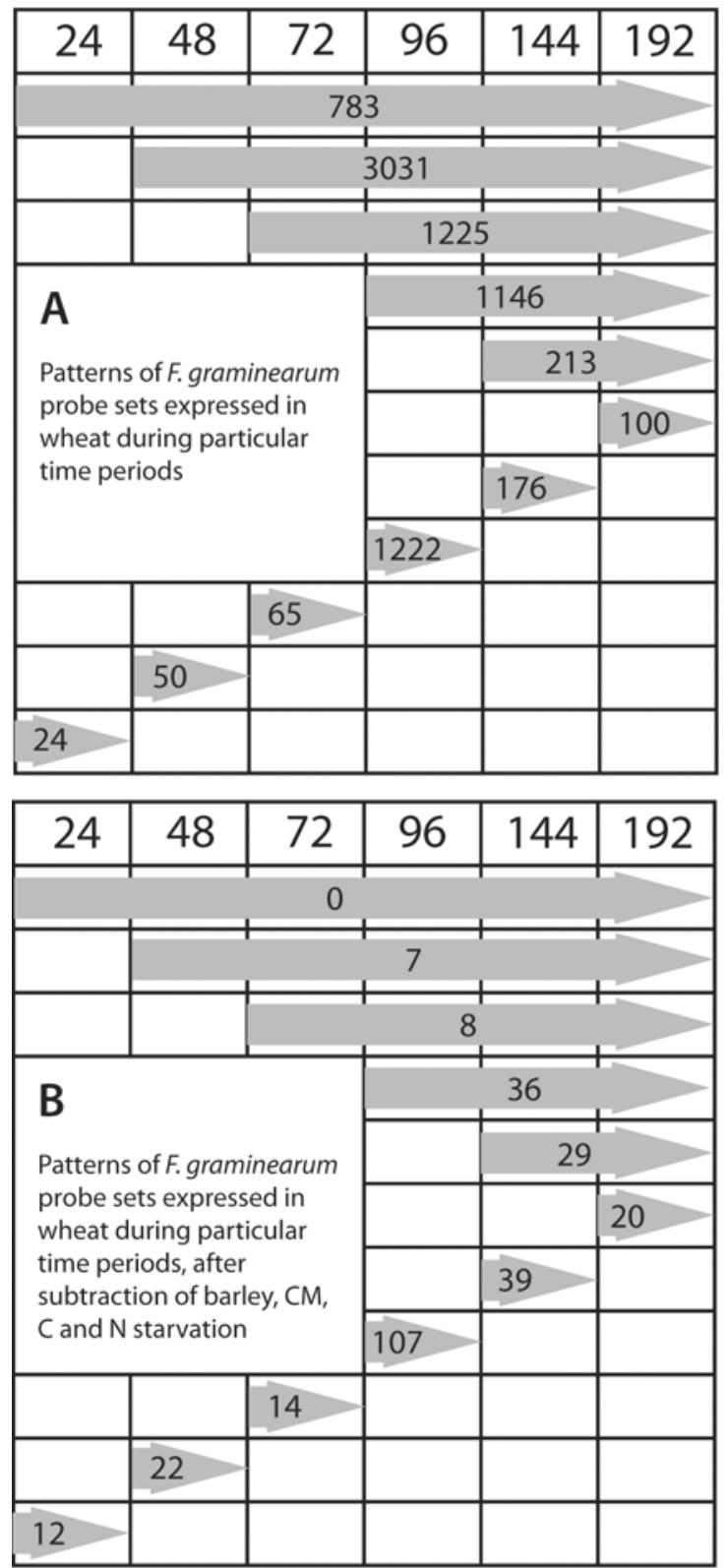

Fig. 4. A, Expression pattern of Fusarium graminearum probe sets in wheat detected during particular periods of time. B, Expression pattern of $F$. graminearum probe sets in wheat detected during particular periods of time, after subtraction of probe sets found expressed in barley, as well as on complete media and $\mathrm{C}$ and $\mathrm{N}$ starvation. 
When we aligned the 355 genes specifically expressed in wheat to the four $F$. graminearum chromosomes, it appeared that they were especially enriched in SNP-rich regions (Fig. 6). To determine whether genes expressed in wheat were more likely to contain SNP than other genes, we compared all 1,950 genes with verified SNP(FG3_FG_SNPs.gff3, Broad Institute, Fusarium Comparative Database) with the 355 wheat-specific genes to determine the proportion having SNP. In fact, $18.9 \%$
(67/355) of genes with wheat-specific expression were determined to have SNP compared with $14.6 \%(1,950 / 13,332)$ for the genome as a whole, a significant enrichment $\left(\chi^{2}, P\right.$ value $=$ $0.02)$. When all in-planta-expressed genes $(n=724)$, except those detected during growth on complete media or $\mathrm{C}$ - and $\mathrm{N}$ starvation, were tested, the proportion of genes with SNP was $18.6 \%$ (135/724), also significantly enriched compared with the genome as a whole $\left(\chi^{2}, P=0.0007\right)$. It appears that in
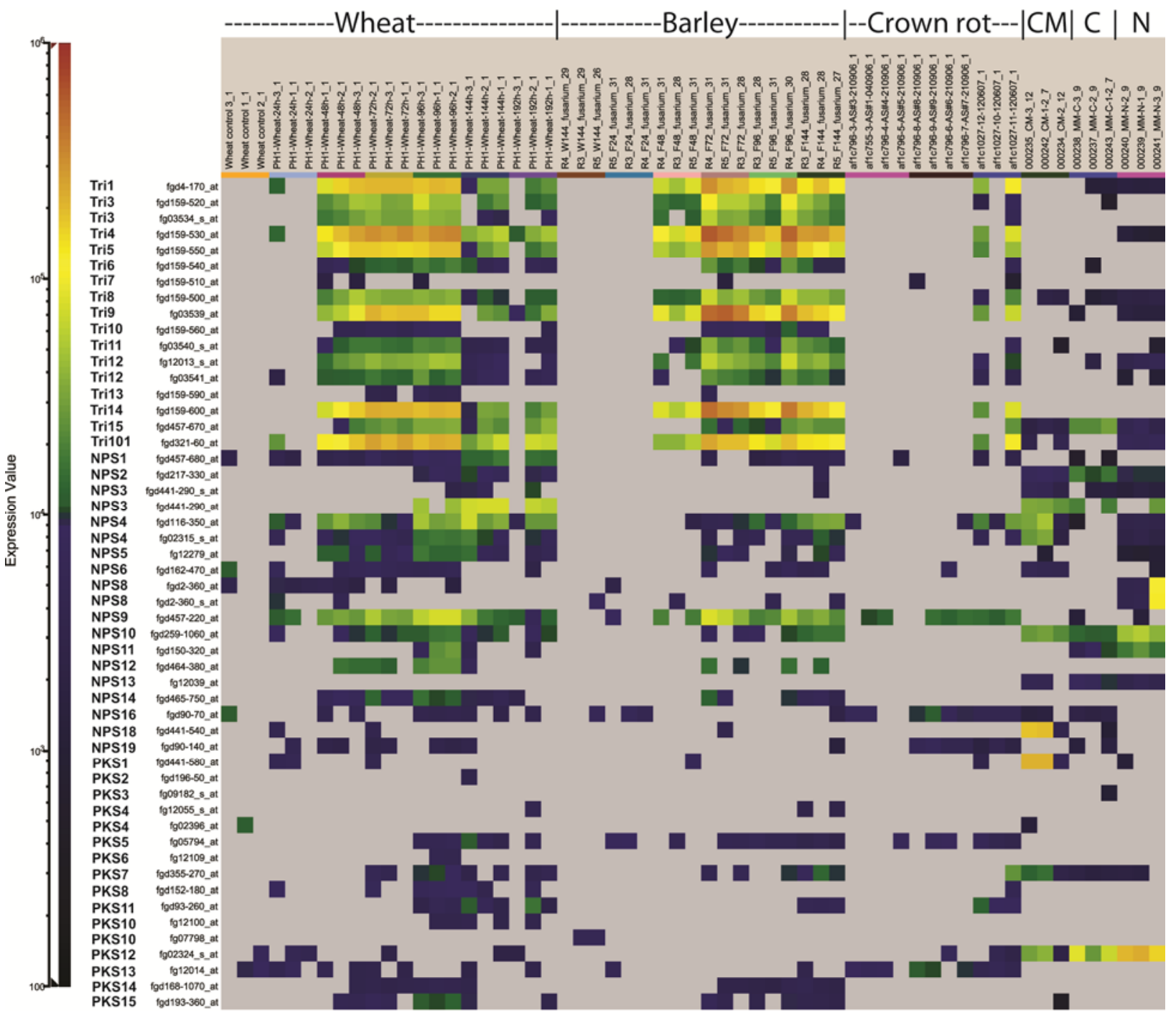

Fig. 5. Expression patterns of secondary metabolite related genes in wheat, barley, and crown rot (Stephens et al. 2008), and on complete media and C and $\mathrm{N}$ starvation. Often, several probe sets for different genes exist, and probe sets with no signal over quality criteria are omitted. Probe sets for NPS7, NPS15, NPS17, and PKS9 did not give any signal under these conditions.
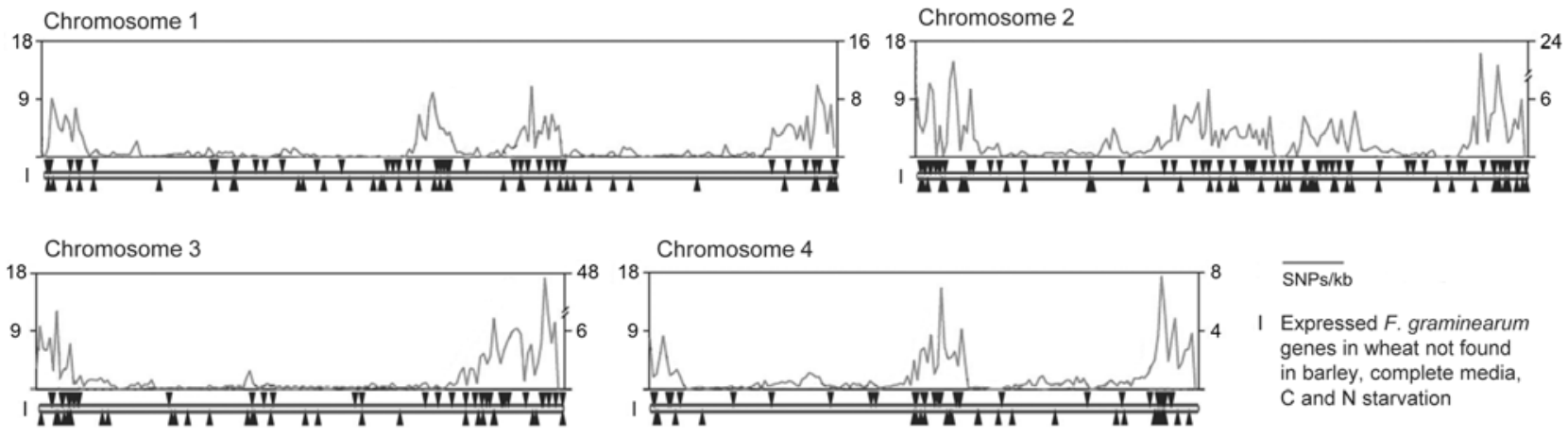

$\overline{\mathrm{SNPs} / \mathrm{kb}}$

Expressed $F$. graminearum genes in wheat not found in barley, complete media, $\mathrm{C}$ and $\mathrm{N}$ starvation

Fig. 6. Alignment of 355 Fusarium graminearum genes specifically expressed in wheat to the four F. graminearum chromosomes. The single-nucleotide polymorphism graph is modified from Cuomo and associates (2007). 
planta-expressed genes are under higher evolutionary selection pressure than expected by chance, and are found in possible pathogenicity islands.

\section{DISCUSSION}

Although the colonization pathway of $F$. graminearum on wheat has been well described, this is the first comprehensive transcriptome study of the infection process. F. graminearum initiates infection at anthesis and the hyphae grow along the epicarp to the space between the lemma and palea, where the epidermis is penetrated (Guenther and Trail 2005; Jansen et al. 2005). In wheat, this is followed by the colonization of the rachis, and movement between the florets of the wheat spike occurs through the vascular bundles. F. graminearum also produces high concentrations of trichothecene toxins during infection of the epicarp, which leads to early cell death and rapid fungal growth through the different layers of the fruit coat (Jansen et al. 2005).

In our studies, an increasing number of probe sets were detected as $F$. graminearum infected wheat heads over time, except for the last two time points (144 and $192 \mathrm{~h}$ ). We attribute the increasing number of probe sets detected and increasing expression levels during the earlier time points as reflecting the increasing biomass of the fungus within the plant tissue. The decreasing level of signal and fewer probe sets detected in the later time points, however, are more difficult to understand. One possible explanation could be due to reduced levels of nutrients in the plant resulting in accumulation of empty fungal hyphae (Klein and Paschke 2004). Another possibility is that, as the plant becomes increasingly necrotic, where most of the host defense mechanisms are defeated, fewer fungal genes may be required. Compared with barley, the wheat infection was more rapid, by as much as $24 \mathrm{~h}$; therefore, a direct comparison of gene expression in wheat and barley for the same time point was not necessarily meaningful. The difference in expression levels between the hosts may be due to different inoculation methods employed; point inoculation was used for wheat while spray inoculation was used for barley. A delay in gene expression for $F$. graminearum in spray- versus pointinoculated plants has been observed by others (L. Harris, personal communication).

The total number of $F$. graminearum probe sets detected in wheat was surprisingly high $(10,007)$ compared with barley $(7,777)$, with substantial overlap with probe sets detected in in vitro experiments including $\mathrm{C}$ starvation (86\%), $\mathrm{N}$ starvation $(86 \%)$, and complete medium $(84 \%)$. As stated previously, the fact that expression of more $F$. graminearum genes were detected in wheat than in barley could be due to differences in the plant inoculation method. However, another possible explanation is that wheat mounts a stronger host defense than barley and, therefore, the fungus requires a greater repertoire of pathogenicity factors to overcome these defenses, hence the higher number of expressed genes. Nevertheless, we found only 355 genes exclusively expressed in wheat, mostly ones with low expression. The majority of these were unclassified protein $(72.6 \%)$, highlighting our limited knowledge of genes involved in the infection process. Most of the genes (11/12) found exclusively in wheat at $24 \mathrm{~h}$ have no known function.

One interesting feature of genes expressed exclusively in wheat was that they were enriched for genes involved in allantoin and allantoate transport $(P=0.001)$. Allantoate permeases were the most enriched genes in this functional category, expressed late during infection. Nitrogen can be a limiting factor for a plant pathogen, and uric acid, the product of diseaseinduced enhanced catabolic processes in the plant, can serve as a fungal nutrient by conversion to allantoin by uricase and subse- quent recovery of ammonia (Divon and Fluhr 2007). The ability to utilize this nitrogen source can be seen as increased pathogen fitness. Genes for allantoin and allantoate transport also were enriched during infection of wheat by a hypervirulent mutant strain of $F$. graminearum (S. Walkowiak, personal communication). The allantoin and allantoate transport category was not enriched (only one gene) among the 369 genes expressed in both wheat and barley, suggesting a mechanism specific to wheat. According to their functional categories, many probe sets detected only in planta correspond to genes predicted to be involved in the breakdown of complex carbon-rich plant substrates and detoxification of host defense molecules, as well as production of secondary metabolites such as the trichothecenes.

When examining the timing of fungal gene expression in wheat (Fig. 4A), 1,222 genes were found only at $96 \mathrm{~h}$, which is approximately 10 times higher than the number specific to any other time point. Of these, 841 were not found in barley at any time point. At approximately 96 h, F. graminearum enters the rachis system in wheat and spreads to neighboring spikelets (Ilgen et al. 2009; Jansen et al. 2005). This spread does not occur in barley, where the fungus is inhibited at the rachis node and rachilla, limiting infection of adjacent florets (Jansen et al. 2005). Some of the genes found specifically expressed in wheat at $96 \mathrm{~h}$ could be involved in achieving this disseminated infection.

Cuomo and associates (2007) suggested that putative pathogenicity factors expressed in barley were found in genomic regions of high genetic diversity. These 45 genes (Cuomo et al. 2007) were also expressed in wheat, suggesting that they could be involved in a common mechanism of infection for both hosts. The genes expressed in both wheat and barley also were highly enriched in secretory pathway signal peptide motifs, supporting their putative role as extracellular virulence factors. Surprisingly, the 355 genes found only in wheat, although located in diversity enriched portions of the genome, were not enriched for signal peptide motifs. Most of the wheat-specific genes had unknown function with low expression levels. However, we speculate that at least some of these genes may have adaptive significance for occupying a nutritional niche in wheat that is not found in barley, or may be wheat-specific pathogenicity factors. Virulence of $F$. graminearum is probably the consequence of timely expression of many genes, governing production of mycotoxins, cell-wall-degrading enzymes, hormones, and specific metabolites that alter the host's resistance reaction (Miedaner 1997). Knowledge of these genes will be valuable for selecting target genes for functional characterization of potential pathogenicity factors.

\section{MATERIALS AND METHODS}

Strains and culture conditions.

The F. graminearum strain PH-1 (NRRL 31084) was used in this study. To produce macroconidia for wheat inoculations, cultures were grown in $100 \mathrm{ml}$ of liquid CMC medium Cappellini and Peterson 1965) at $25^{\circ} \mathrm{C}$, in light and with shaking at $150 \mathrm{rpm}$ for $24 \mathrm{~h}$. The cultures were filtered through Miracloth and centrifuged to collect macroconidia.

\section{Wheat infection time course.}

Wheat (Triticum aestivum) 'Bobwhite' was grown as previously described (Goswami and Kistler 2005). All spikelets were point inoculated (injecting a spore suspension into individual florets) at anthesis with $10 \mu \mathrm{l}$ of conidial suspension of PH-1 ( $10^{5}$ spores $\left./ \mathrm{ml}\right)$ or water control (mock) in $0.01 \%$ Triton 60 solution. After inoculation, the plants were placed in a growth chamber at $16^{\circ} \mathrm{C}$ for $8 \mathrm{~h}$ (night) and $18^{\circ} \mathrm{C}$ for $16 \mathrm{~h}$ (day). To ensure proper disease severity, the spikes were cov- 
ered with plastic bags for $48 \mathrm{~h}$ to increase the humidity. Three biological replicates were conducted at 24, 48, 72, 96, 144, and 192 hai. Water-treated (mock) spikes from the 0-h time point was used as a control. For each biological replication, four spikes were randomly sampled and immediately frozen at $-80^{\circ} \mathrm{C}$ prior to RNA extraction. At each of the time points, spikes were sampled at approximately 3:00 p.m.

\section{Microarray experiments.}

Total RNA was isolated using TRIzol reagent (Invitrogen, Carlsbad, CA, U.S.A.) and purified with an RNeasy Mini kit (Qiagen, Valencia, CA, U.S.A.) according to the manufacturers' instructions. Total RNA $(10 \mu \mathrm{g})$ was labeled according to Affymetrix eukaryotic RNA-labeling protocols (Affymetrix, Santa Clara, CA, U.S.A.). The labeled RNA was hybridized with $F$. graminearum Affymetrix GeneChip (Guldener et al. 2006). The processing and data acquisition from chips followed standard Affymetrix procedures in use at the Biomedical Image Processing Facility at the University of Minnesota. The resulting CEL files were analyzed with Genedata Expressionist Software. The CEL files were normalized using the RMA algorithm and all experiments were performed at $P=0.04$. A $t$ test was used to analyze differential expression, and a present-or-absent test was performed, where "present" indicated significant signal from the probe sets above background in at least two of the three biological replicate chips and "absent" indicated signal not significantly above background in at least two out of three chips. MIPS FunCat was used to analyze functional categories from differentially expressed genes (Ruepp et al. 2004). For the similarity dendrogram, data were RMA normalized using R, Bioconductor (Gentleman et al. 2004), and the "affy" package. Data from the microarray experiments are stored as experiment FG15 at PLEXdb (Wise et al. 2006).

\section{ACKNOWLEDGMENTS}

We thank W. Xu and the University of Minnesota, Supercomputing Institute for advice and computing resources that made this study possible; K. Broz (United States Department of Agriculture-Agricultural Research Service Cereal Disease Laboratory) for outstanding technical assistance; S. S. Klemsdal (Bioforsk) for support; and U. Guldener (Institute of Bioinformatics and Systems Biology, German Research Center for Environmental Health) for assistance with MIPS FunCat analysis. This project was supported by the National Research Initiative of the United States Department of Agriculture National Institute of Food and Agriculture, grant 2004-35604-14327, and the BILAT project 173277 supported by the Research Council of Norway.

\section{LITERATURE CITED}

Cappellini, R. A., and Peterson, J. L. 1965. Macroconidium formation in submerged cultures by a non-sporulating strain of Gibberella zeae. 962966.

Cuomo, C. A., Guldener, U., Xu, J. R., Trail, F., Turgeon, B. G., Di Pietro, A., Walton,, J. D., Ma, L. J., Baker, S. E., Rep, M., Adam, G., Antoniw, J., Baldwin, T., Calvo, S., Chang, Y.L., Decaprio, D., Gale, L. R., Gnerre, S., Goswami, R. S., Hammond-Kosack, K., Harris, L. J., Hilburn, K., Kennell, J. C., Kroken, S., Magnuson, J. K., Mannhaupt, G., Mauceli, E., Mewes, H. W., Mitterbauer, R., Muehlbauer, G., Münsterkötter, M., Nelson, D., O’donnell, K., Ouellet, T., Qi, W., Quesneville, H., Roncero, M. I., Seong, K. Y., Tetko, I. V., Urban, M., Waalwijk, C., Ward, T. J., Yao, J., Birren, B. W., and Kistler, H. C. 2007. The Fusarium graminearum genome reveals a link between localized polymorphism and pathogen specialization. Science 317:1400-1402.
Divon, H. H., and Fluhr, R. 2007. Nutrition acquisition strategies during fungal infection of plants. FEMS (Fed. Eur. Microbiol. Soc.) Microbiol. Lett. 266:65-74.

Gentleman, R. C., Carey, V. J., Bates, D. M., Bolstad, B., Dettling, M., Dudoit, S., Ellis, B. et al. 2004. Bioconductor: Open software development for computational biology and bioinformatics. Genome Biol. 5.

Goswami, R. S., and Kistler, H. C. 2004. Heading for disaster: Fusarium graminearum on cereal crops. Mol. Plant Pathol. 5:515-525.

Goswami, R. S., and Kistler, H. C. 2005. Pathogenicity and in planta mycotoxin accumulation among members of the Fusarium graminearum species complex on wheat and rice. Phytopathology 95:1397-1404.

Guenther, J. C., and Trail, F. 2005. The development and differentiation of Gibberella zeae (anamorph: Fusarium graminearum) during colonization of wheat. Mycologia 97:229-237.

Guldener, U., Seong, K. Y., Boddu, J., Cho, S., Trail, F., Xu, J. R., Adam, G. et al. 2006. Development of a Fusarium graminearum Affymetrix GeneChip for profiling fungal gene expression in vitro and in planta. Fungal Genet. Biol. 43:316-325.

Ilgen, P., Hadeler, B., Maier, F. J., and Schafer, W. 2009. Developing kernel and rachis node induce the trichothecene pathway of Fusarium graminearum during wheat head infection. Mol. Plant-Microbe Interact. 22:899-908.

Jansen, C., von Wettstein, D., Schafer, W., Kogel, K. H., Felk, A., and Maier, F. J. 2005. Infection patterns in barley and wheat spikes inoculated with wild-type and trichodiene synthase gene disrupted Fusarium graminearum. Proc. Natl. Acad. Sci. U.S.A. 102:16892-16897.

Klein, D. A., and Paschke, M. W.. 2004. Filamentous fungi: The indeterminate lifestyle and microbial ecology. Microb. Ecol. 47:224-235.

Lauren, D. R., and Smith, W. A. 2001. Stability of the Fusarium mycotoxins nivalenol, deoxynivalenol and zearalenone in ground maize under typical cooking environments. Food Addit. Contam. 18:10111016.

Ma, L. J., van der Does, H. C., Borkovich, K. A., Coleman, J. J., Daboussi, M. J., Di Pietro, A., Dufresne, M. et al. 2010. Comparative genomics reveals mobile pathogenicity chromosomes in Fusarium. Nature 464:367-373.

McMullen, M., Jones, R., and Gallenberg, D. 1997. Scab of wheat and barley: A re-emerging disease of devastating impact. Plant Dis. 81:13401348.

Mewes, H. W., Amid, C., Arnold, R., Frishman, D., Guldener, U. Mannhaupt, G., Munsterkotter, M. et al. 2004. MIPS: Analysis and annotation of proteins from whole genomes. Nucleic Acids Res. 32:D41-D44

Miedaner, T. 1997. Breeding wheat and rye for resistance to Fusarium diseases. Plant Breed. 116:201-220.

O’Donnell, K., Ward, T. J., Geiser, D. M., Kistler, H. C., and Aoki, T. 2004. Genealogical concordance between the mating type locus and seven other nuclear genes supports formal recognition of nine phylogenetically distinct species within the Fusarium graminearum clade. Fungal Genet. Biol. 41:600-623.

Paper, J. M., Scott-Craig, J. S., Adhikari, N. D., Cuomo, C. A., and Walton, J. D. 2007. Comparative proteomics of extracellular proteins in vitro and in planta from the pathogenic fungus Fusarium graminearum. Proteomics 7:3171-3183.

Ruepp, A., Zollner, A., Maier, D., Albermann, K., Hani, J., Mokrejs, M., Tetko, I. et al. 2004. The FunCat, a functional annotation scheme for systematic classification of proteins from whole genomes. Nucleic Acids Res. 32:5539-5545.

Stephens, A. E., Gardiner, D. M., White, R. G., Munn, A. L., and Manners, J. M. 2008. Phases of infection and gene expression of Fusarium graminearum during crown rot disease of wheat. Mol. Plant-Microbe Interact. 21:1571-1581.

Wise, R., Caldo, R., Hong, L., Wu, S., Cannon, E., and Dickerson, J. 2006. PLEXdb: A unified expression profiling database for plants and plant pathogens. (Abstr.) Phytopathology 96:S161.

\section{AUTHOR-RECOMMENDED INTERNET RESOURCES}

The Comprehensive R Archive Network website: cran.r-project.org Genedata Expressionist software: www.genedata.com PLEXdb database: www.plexdb.org 